\title{
An Embedded Boundary Cartesian Grid Scheme for Viscous Flows using a New Viscous Wall Boundary Condition Treatment
}

\author{
David D. Marshall * and Stephen M. Ruffin ${ }^{\dagger}$ \\ School of Aerospace Engineering \\ Georgia Institute of Technology \\ Atlanta, GA 30332-0150
}

\begin{abstract}
This work presents a new viscous wall boundary condition technique for embedded Cartesian grid schemes in order to model laminar viscous flows. The development of viscous effects modeling using pure Cartesian grids with cut cells at the surface has been hampered by the widely varying control volume sizes associated with the mesh refinement and the cut cells associated with the solid surface. This scheme removes the cells adjacent to the surface from the control volume formulation. These cells are instead solved via an interpolation technique which utilizes the wall boundary conditions to build the interpolating functions. Two different interpolation techniques are presented, one without considering wall curvature and one considering wall curvature. Results are compared to subsonic and supersonic two-dimensional airfoil cases.
\end{abstract}

Nomenclature

$C_{d} \quad$ Drag coefficient

$C_{f} \quad$ Skin friction coefficient

$C_{l} \quad$ Lift coefficient

$C_{p} \quad$ Pressure coefficient

$c_{p}$

$C F L$

$K_{w}$

$K_{\eta \xi}$

$K_{\eta \zeta}$

$M$

n

$\operatorname{Pr}$

$p$

$R$

Re

$T$

$\boldsymbol{u}$

$u$

$x, y, z$

$\gamma$

$\delta$

$\lambda$

$\kappa$

$\mu$

$\xi, \eta, \zeta$

Specific heat at constant pressure

Courant-Friedrichs-Levy condition number

Wall curvature constant combining curvatures and tangential velocity direction

Curvature of the surface along the $\xi$-direction

Curvature of the surface along the $\zeta$-direction

Mach number

Normal vector from surface

Prandtl Number

Pressure

Gas constant

Reynolds number

Temperature

Velocity vector

Velocity vector component

Cartesian coordinate directions

Specific heat ratio

Distance along surface normal

Velocity direction in the $\xi-\zeta$ plane

Thermal conductivity

Dynamics viscosity

Geodesic coordinate system for surface

curvature formulation

Subscripts

$\xi, \eta, \zeta \quad$ Vector component in given geodesic coordinate direction

\footnotetext{
${ }^{*}$ Sr. Engineer, Advatech Pacifi c Inc., Edwards AFB, CA, Member AIAA

$\dagger$ Associate Professor, Member AIAA
}

$\begin{array}{ll}c & \begin{array}{l}\text { Property at interpolation point along } \\ \text { surface normal }\end{array} \\ t & \text { Vector property tangential to wall } \\ w & \text { Property at wall } \\ 0 & \text { Stagnation properties } \\ 9 & \text { Surface cell centroid property in wall } \\ & \text { boundary condition development } \\ \infty & \text { Freestream conditions }\end{array}$

\section{Introduction}

CARTESIAN grids have been gaining popularity in the past several years because of the short grid generation times required for complex three dimensional configurations. ${ }^{1}$ This has motivated a number of researchers to develop inviscid, embedded grid Cartesian solvers that utilize the cut cell methodology to model the surfaces. ${ }^{2-4}$

While this has provided excellent results for inviscid solutions, Coirier and Powell ${ }^{5}$ demonstrated that extreme oscillations result near the cut cells for even simple geometries due to the non-positivity of the stencils used in several viscous flux reconstruction techniques. They demonstrated that all of the schemes they studied demonstrated (to some degree) a competition between the accuracy of the scheme and the viscous stencil positivity for non-uniform cells, i.e. any attempt to improve the accuracy/positivity adversely effected the resulting positivity/accuracy.

While Coirier and Powell demonstrated excellent agreement with their Euler Cartesian grid solver, even simple flat plate Blasius configurations proved difficult to accurately capture when there were cut cells in the computational domain. Coirier's results for a Blasius flat plate configuration with the plate at an angle of $30^{\circ}$ with respect to the $x$-axis show large oscillations in the skin friction coefficient. This non-smoothness problem 
was also observed in Frymier's work, ${ }^{6}$ but it was not attributed to the viscous stencil positivity. Thus, if general bodies need to be modeled for viscous solutions, then these solid surface boundary condition formulations are of little use.

In addition to the non-smoothness problems associated with the existing solid boundary treatment, the cut cells generated by the solid surface intersecting with the Cartesian cells require very small time steps to maintain the CFL restriction needed to ensure the stability of explicit time integration schemes. Thus, removing the surface cells from the finite volume formulation will provide the added benefit of relaxing the time step restriction.

\section{Cartesian Solver Description}

The Cartesian grid solver used for this work is NASCART-GT, an unsteady, three-dimensional solver developed at Georgia Tech. A summary of the features will be presented here, see Marshall ${ }^{7}$ for a detailed description.

NASCART-GT is an unsteady, three-dimensional embedded grid Cartesian solver of the full laminar NavierStokes equations without body forces and a perfect gas thermodynamic model. The governing equations are solved using Roe's approximate Riemann solver coupled with a MUSCL data reconstruction technique for the inviscid fluxes and traditional finite differencing of the viscous terms. A pressure based limiter is applied to the reconstruction to dampen oscillations in high gradient regions. The overall accuracy of the scheme is as high as second order accurate in space.

The time integration is performed using a Hancock two-stage predictor-corrector scheme which is second order accurate in time. In order to accurately capture high gradient regions, a solution adaption scheme is used. The solution adaption scheme uses a combination of the velocity divergence and velocity gradient as a coarsening/refining metric.

Since no ghost-cells are used at the surface cells, the MUSCL data reconstruction limiter is used to avoid creating a computational stencil with cells that are interior to the body. This has the effect of possibly lowering the order of accuracy of the data reconstruction for some cells near the surface.

\section{Wall Boundary Condition Development}

The wall boundary condition development presented here removes the surface cells from the finite volume formulation discussed above. Instead, the state for these cells is determined from an interpolation of the surrounding cells with the wall boundary conditions as additional constraints. Notice that this treatment removes the arbitrarily small cut cells from the finite volume formulation and increases the allowable time step size, a side benefit explored in another paper. ${ }^{8}$
The formulation of the surface cell properties utilizes the state at a point normal to the surface which can be based on the surrounding cells, see figure 1 . The state at point 'c' is constructed either directly from the state of the cell containing point 'c' (in this case labeled '5'), or by using a distance weighted interpolation of the of the surrounding cells (in this case cells '1' through '9'). The distance weighted interpolation places a restriction on the cells surrounding the surface cell such that all of the cells neighboring the reference cell and the reference cell itself must be at the same refinement level as the surface cell.

Using the state at point 'c', the state at the centroid of the surface cell, labeled '9', (or the wall location, labeled ' $w$ ') can be developed by using one-dimensional relationships along the line $\overline{B w}$. The specifics of the state reconstruction depends on whether the wall curvature is to be considered.

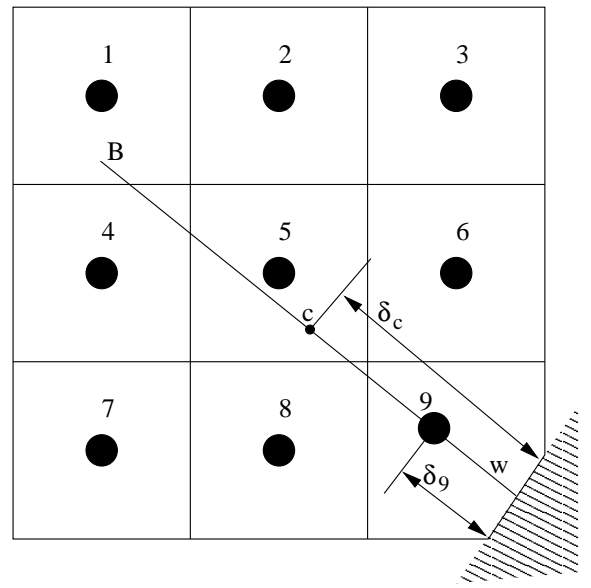

Fig. 1 Example Confi guration for Solid Boundary Treatment

Flat Wall Development

The viscous formulation is separated into two cases, one if the flow at point 'c' is subsonic and another if it is supersonic.

Subsonic Case The surface cell velocity is first determined by an interpolation procedure along the line $\overline{B w}$ from point 'c' to the wall utilizing the no slip wall boundary condition. The resulting relationship is

$$
\boldsymbol{u}_{9}=\left[\boldsymbol{u}_{C}-\left(1-\frac{\delta_{9}}{\delta_{C}}\right)\left(\boldsymbol{u}_{C} \cdot \boldsymbol{n}\right) \boldsymbol{n}\right]\left(\frac{\delta_{9}}{\delta_{C}}\right)
$$

where $\delta_{c}$ and $\delta_{9}$ are the distances from point ' $w$ ' to points 'c' and '9', respectively. This has the effect of linearly decreasing the tangential velocity to zero and quadratically decreasing the normal velocity to zero at the wall.

Next, the pressure at point '9' can be determined by using the normal momentum equation for a flat wall to get

$$
\frac{d p}{d n}=0
$$


which when used in a first order forward finite difference approximation yields

$$
p_{9}=p_{c}
$$

To close the thermodynamic system and enforce the final wall boundary condition, the temperature for the surface cell is determined. For an adiabatic wall boundary condition, a first order finite difference formulation for the wall heat flux yields the simple relation

$$
T_{9}=T_{c}
$$

While for the isothermal case, a simple linear interpolation along $\overline{B w}$, yields

$$
T_{9}=\frac{\delta_{9}}{\delta_{c}} T_{c}+\left(1-\frac{\delta_{9}}{\delta_{c}}\right) T_{w}
$$

Supersonic Case The supersonic case should be a pathological case since the wall cell must be in the boundary layer (thus subsonic), but it is applicable when the solution domain is initialized using the freestream values. If the wall angle produces a shock then the subsonic viscous velocity formulation is used to determine the velocity direction and the oblique shock relations are used to calculate the velocity magnitude and the thermodynamic conditions.

\section{Curved Wall Development}

While the basic model does address many of the problems with the viscous flux stencil positivity mentioned above, an enhanced version of the wall boundary conditions is presented in the curved wall model. Specifically, utilizing the surface curvature in an effort to ease the grid refinement criteria around regions of high curvature, and utilizing the governing equations to develop the interpolation relationships. The surface curvature modification requires the governing equations to be transformed into geodesic coordinates in order to incorporate the surface curvature terms. See Marshall ${ }^{7}$ for the derivation details associated with the full NavierStokes and the boundary layer equations in both twoand three-dimensions for geodesic coordinates. The net result of this treatment is that the surface curvature is needed along the two coordinates on the surface ( $\xi$ and $\zeta$ ). The curvature of the surface along the $\xi$-direction is denoted as $K_{\eta \xi}$ and along the $\zeta$-direction is denoted as $K_{\eta \zeta}$. See figure 2 for an example surface.

There are two important equations that will be used in this work, the normal momentum equation and the energy equation. The normal momentum equation is used to improve the pressure determination into a quadratic interpolation, and the energy equation is used to improve the temperature determination into a quadratic interpolation.

The normal momentum equation in the geodesic for-

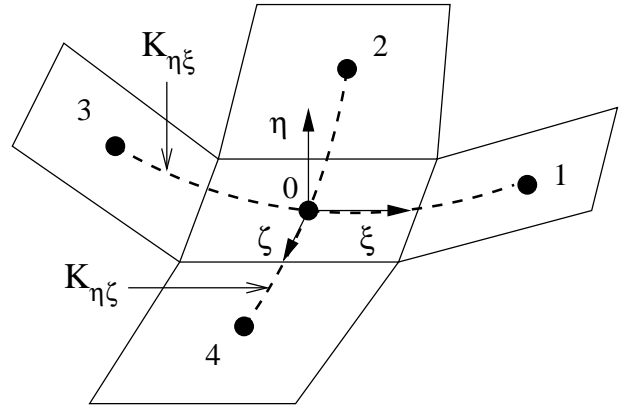

Fig. 2 Example Surface for Curvature Calculation

mulation is

$$
\begin{aligned}
\frac{\partial p}{\partial \eta} & =\rho\left[K_{\eta \xi} u_{\xi}^{2}+K_{\eta \zeta} u_{\zeta}^{2}\right] \\
& =\rho u_{t}^{2}\left[K_{\eta \xi} \cos ^{2} \lambda+K_{\eta \zeta} \sin ^{2} \lambda\right]
\end{aligned}
$$

where $u_{t}$ is the tangential component of the velocity vector. Unlike the inviscid formulation of the normal momentum equation, this equation holds throughout the boundary layer and not just at the wall, as long as the curvatures are evaluated at the point in the boundary layer and not on the surface.

The energy equation in the geodesic formulation is

$$
\begin{aligned}
\rho \frac{\partial H}{\partial t}+ & \rho\left[\left(\frac{1}{h_{\xi}}\right) u_{\xi} \frac{\partial H}{\partial \xi}+u_{\eta} \frac{\partial H}{\partial \eta}+\left(\frac{1}{h_{\zeta}}\right) u_{\zeta} \frac{\partial H}{\partial \zeta}\right] \\
= & \frac{\partial p}{\partial t}+\frac{\partial Q}{\partial t}+\frac{\partial}{\partial \eta}\left[\frac{\mu}{\operatorname{Pr}} \frac{\partial H}{\partial \eta}\right] \\
& +\frac{\partial}{\partial \eta}\left[\mu\left(1-\frac{1}{P r}\right)\left(u_{\xi} \frac{\partial u_{\xi}}{\partial \eta}+u_{\zeta} \frac{\partial u_{\zeta}}{\partial \eta}\right)\right]
\end{aligned}
$$

If steady state is assumed and the equation is applied to the wall (where $u=0$ ), then equation (7) becomes

$$
\begin{aligned}
\frac{\partial}{\partial \eta} & {\left[\frac{\mu}{\operatorname{Pr}} \frac{\partial H}{\partial \eta}\right]_{w} } \\
& +\frac{\partial}{\partial \eta}\left[\mu\left(1-\frac{1}{\operatorname{Pr}}\right)\left(u_{\xi} \frac{\partial u_{\xi}}{\partial \eta}+u_{\zeta} \frac{\partial u_{\zeta}}{\partial \eta}\right)\right]_{w}=0
\end{aligned}
$$

where all derivatives are taken at the wall. Converting this from stagnation enthalpy to temperature, $H=$ $C_{p} T+U^{2} / 2$, and recalling the constant specific heats assumption of NASCART-GT yields as well as the boundary layer assumptions that the tangential velocity is much larger than the normal velocity (i.e. $u_{t} \gg u_{\eta}$ ), the linear velocity profile assumption yields

$$
\left.\frac{\partial^{2} T}{\partial \eta^{2}}\right|_{w}+\frac{P r}{C_{p}}\left(\left.\frac{\partial u_{t}}{\partial \eta}\right|_{w}\right)^{2}+\left.\left.\frac{1}{\mu_{w}} \frac{\partial \mu}{\partial \eta}\right|_{w} \frac{\partial T}{\partial \eta}\right|_{w}=0
$$

Finally, if the assumption of constant viscosity at the wall is used then the boundary layer energy equation at the wall becomes

$$
\left.\frac{\partial^{2} T}{\partial \eta^{2}}\right|_{w}=-\frac{\operatorname{Pr}}{C_{p}}\left(\left.\frac{\partial u_{t}}{\partial \eta}\right|_{w}\right)^{2}
$$


As with the flat wall boundary conditions, the viscous formulation is separated into two cases, one if the flow at point ' $c$ ' is subsonic and another if it is supersonic.

Subsonic Case The subsonic viscous wall conditions start with an assumption of the velocity profiles. The direction of the tangential velocity is assumed constant. Since there are only two conditions available to build a velocity profile around, the velocity at point 'c' and the no-slip boundary condition at the wall, the velocity profiles are limited to linear profiles defined as

$$
\begin{aligned}
u_{\eta} & =\frac{\delta}{\delta_{c}} u_{\eta, c} \\
u_{t} & =\frac{\delta}{\delta_{c}} u_{t, c}
\end{aligned}
$$

For the pressure boundary condition there are three conditions known, the pressure at point 'c' and $\frac{\partial p}{\partial \eta}$ at the wall as well as point ' $c$ ' from the boundary layer equations in geodesic coordinates presented above. Applying the normal momentum equation of the boundary layer equation (6) to these conditions yields

$$
\begin{aligned}
\left.\frac{\partial p}{\partial \eta}\right|_{w} & =0 \\
\left.\frac{\partial p}{\partial \eta}\right|_{c} & =\rho_{c} u_{t, c}^{2} K_{c} \\
K_{c} & =K_{\eta \xi, c} \cos ^{2} \lambda_{c}+K_{\eta \zeta, c} \sin ^{2} \lambda_{c}
\end{aligned}
$$

where $K_{c}$ is the combined curvature effects in the $\xi$ and $\zeta$ directions at point ' $c$ ' (not at the wall). With three conditions a quadratic profile can be used to describe the pressure distribution throughout the boundary layer to get

$$
p=\frac{1}{2}\left(\rho_{c} u_{t, c}^{2} K_{c} \delta_{c}\right)\left[\left(\frac{\delta}{\delta_{c}}\right)^{2}-1\right]+p_{c}
$$

The development of the final condition, temperature, utilizes the compressible boundary layer energy equation in geodesic coordinates presented above. This equation along with the known state at point 'c' and the wall thermal boundary condition (either adiabatic or isothermal wall) yield three conditions. Therefore a quadratic profile can be used to describe the temperature distribution throughout the boundary layer for the adiabatic wall boundary condition, $\left.\frac{\partial T}{\partial \eta}\right|_{w}=0$, which results in the following:

$$
T=-\frac{\operatorname{Pr}}{2 C_{p}} u_{t, c}^{2}\left[\left(\frac{\delta}{\delta_{c}}\right)^{2}-1\right]+T_{c}
$$

For the isothermal wall boundary condition, $T_{w}$ is specified, which results in the following equation for the temperature profile

$$
\begin{aligned}
T & =-\frac{\operatorname{Pr}}{2 C_{p}} u_{t, c}^{2}\left[\left(\frac{\delta}{\delta_{c}}\right)^{2}-\left(\frac{\delta}{\delta_{c}}\right)\right] \\
& +\left(T_{c}-T_{w}\right) \frac{\delta}{\delta_{c}}+T_{w}
\end{aligned}
$$

Supersonic Case As with the basic model, this pathological case is treated by using the oblique shock relations from above to calculate the velocity magnitude and the thermodynamic conditions, while the velocity direction is obtained from the subsonic formulation.

\section{Results}

The following cases demonstrate the effectiveness of the new viscous wall boundary conditions presented above. The first case is a subsonic NACA-0012 airfoil flow. This is followed by a supersonic NACA-0012 airfoil flow.

\section{Subsonic NACA-0012 Airfoil}

This test case is a NACA-0012 airfoil in a $M_{\infty}=0.8$ flow at an angle-of-attack of $\alpha=10^{\circ}$ and a freestream Reynolds number of $R e_{\infty}=500$. The computational boundaries are approximately 10 chords around the airfoil. Solutions are presented on a computational domain with a root grid dimension of $240 \times 216$ and 4 levels of refinement. In addition, solution adaption is performed every 2000 iterations starting after 1000 iterations. The final grids for the flat wall solution consists of 105,565 cells and 105,535 cells for the curved wall solution. Also, a curvature maximum of $\mathbf{4 0 . 0}$ is imposed in order to limit the large pressure gradients that can result near the leading edge. Figure 3 shows the final grid for the curved wall solution.

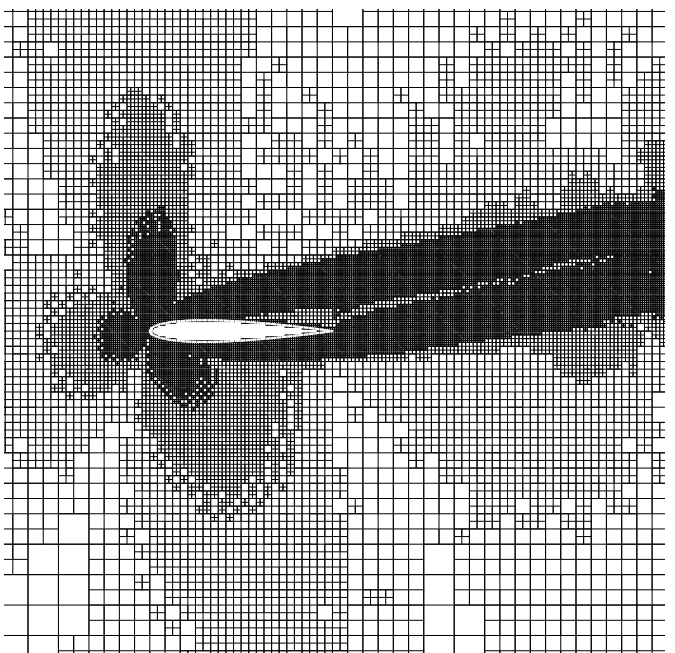

Fig. 3 Final Computational Domain for Subsonic NACA0012 Flow

The results from this case are compared with the results from Casalini and Dadone, ${ }^{9}$ whose results compare quite well to a collection of results from Bristeau et 
al. ${ }^{10}$ from an international workshop on compressible Navier-Stokes solvers. The Casalini and Dadone results are from a structured grid solution with $256 \times 64(16,384)$ cells.

Figure 4 shows the surface pressure coefficient comparison between the NASCART-GT solutions and the results from Casalini and Dadone. The flat wall and curved wall solutions show little differences between each other. They both capture the suction peak near the leading edge reasonably well, and slightly over-predict the lower surface pressure. In general, the agreement between the reference solution and the NASCART-GT surface pressure coefficient distributions is good.

Figure 5 shows the skin friction coefficient comparison between the NASCART-GT solutions and the results from Casalini and Dadone. Here, the leading edge skin friction coefficient is not well resolved until $x / L$ of 0.1 on the upper surface and 0.15 on the lower surface. This is simply a grid resolution problem that would require multiple levels of grid cells along the body to reasonably capture the leading edge effects, which is currently not an option in NASCART-GT. Adding this functionality would require careful examination of the viscous stencil positivity criteria discussed by Coirier and Powell ${ }^{5}$ in order to ensure that non-smoothness is not introduced into the solution. Notice that there are no large oscillations in the skin friction coefficient as was shown by other cut cell Cartesian approaches. Generally, after the leading edge resolution problem, there is excellent agreement between the reference skin friction coefficient and the NASCART-GT solutions.

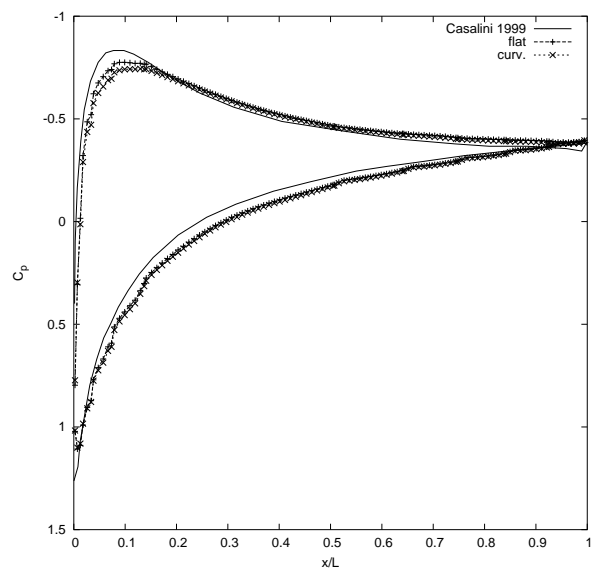

Fig. 4 Subsonic Viscous NACA-0012 Surface Pressure Coeffi cient

Figures 6 and 7 show the Mach contours for the flat wall and curved wall solutions, respectively. Figure 8 shows the Mach contours from the Casalini and Dadone reference. All three figures use a $\Delta M=0.05$ for the contours. Both wall boundary conditions do an excellent job of capturing the flow features throughout the computational domain. In particular the recirculation region is clearly evident in both solutions.

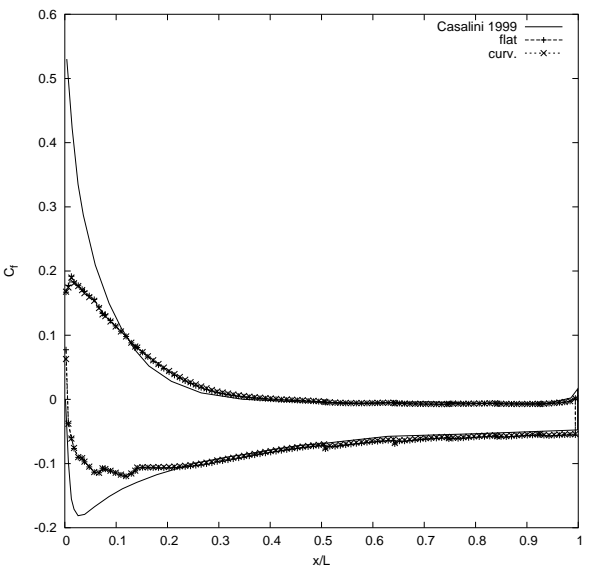

Fig. 5 Subsonic Viscous NACA-0012 Skin Friction Coeffi cient

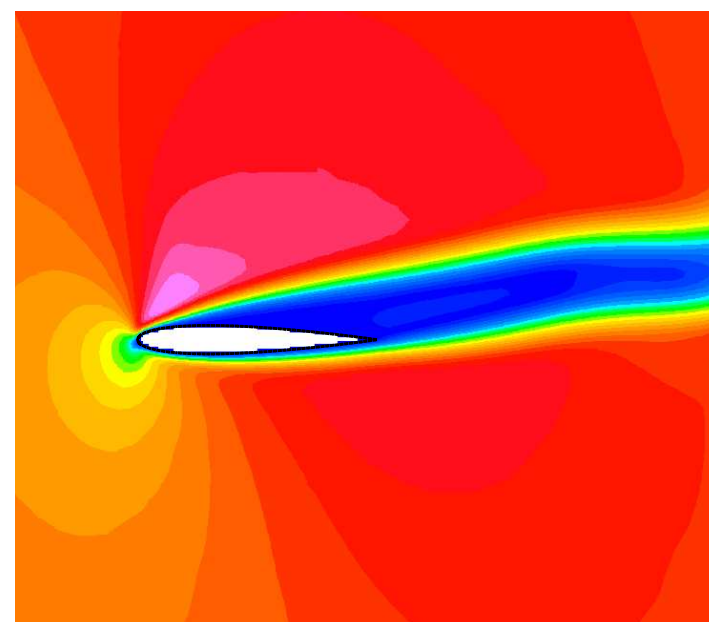

Fig. 6 Mach Contours for Subsonic Viscous NACA-0012 Flow Flat Wall

Finally, table 1 shows the lift and drag coefficients as well as the separation point for the flat wall and curved wall cases. These results are again compared to the Casalini and Dadone references mentioned above. The flat wall boundary condition over predicts the lift coefficient by $5.1 \%$ and slightly under predicts the drag coefficient by $1.6 \%$. The curved wall boundary condition also over predicts the the lift coefficient by $5.6 \%$ and slightly over predicts the drag coefficient by $2.0 \%$. An examination of the skin friction coefficients for both solutions shows that the separation point occurs around $x / L$ of 0.41 for the flat wall and curved wall solutions, which is 0.08 chords off of the location from Casalini and Dadone of 0.33 .

\begin{tabular}{cccc}
\hline & flat wall & curved wall & Casalini \& Dadone $^{9}$ \\
\hline$C_{l}$ & 0.413 & 0.415 & 0.393 \\
$C_{d}$ & 0.249 & 0.248 & 0.253 \\
$x_{\text {sep }} / L$ & 0.41 & 0.41 & 0.33 \\
\hline
\end{tabular}

Table 1 Subsonic Viscous NACA-0012 Lift and Drag Results 


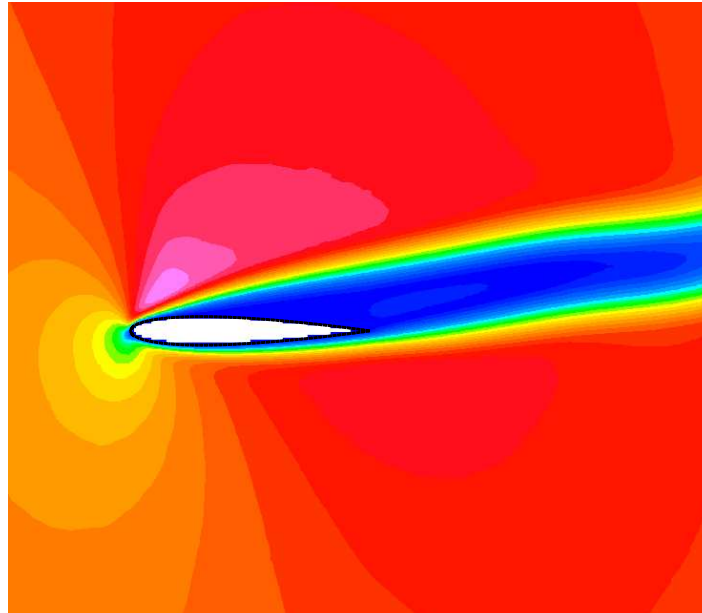

Fig. 7 Mach Contours for Subsonic Viscous NACA-0012 Flow Curved Wall

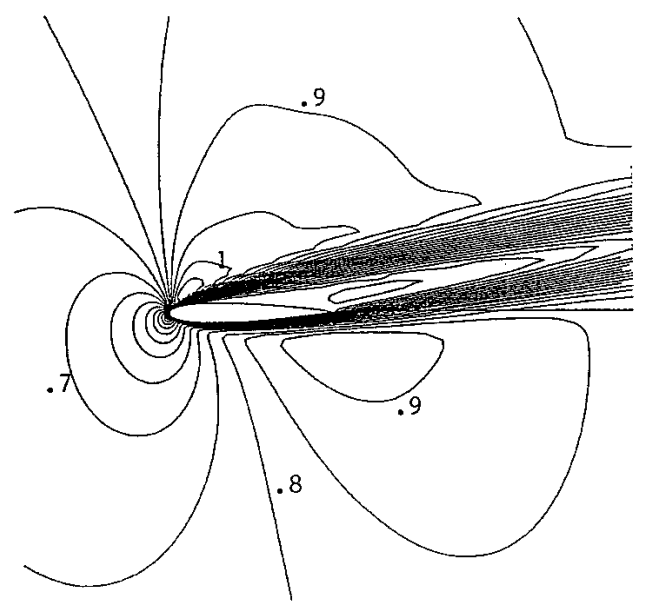

Fig. 8 Viscous Subsonic NACA-0012 Mach Contours from Casalini and Dadone ${ }^{9}$

\section{Supersonic NACA-0012 Airfoil}

This test case is a NACA-0012 airfoil in a $M_{\infty}=2.0$ flow at an angle-of-attack of $\alpha=10^{\circ}$ and a freestream Reynolds number of $R e_{\infty}=1000$. The computational boundaries are 1 chord ahead of the airfoil, 6 chords behind the airfoil and 5 chords above and 3 chords below the airfoil centerline. Solutions are presented on a computational domain with a root grid dimension of $256 \times 256$ and 4 levels of refinement. In addition, solution adaption is performed every 5000 iterations starting after 1000 iterations. The final grids for the flat wall solution consists of $\mathbf{1 5 8 , 8 4 2}$ cells and 158,947 cells for the curved wall solution. Also, a curvature maximum of 40.0 is imposed in order to limit the pressure gradients caused by the highly curved regions of the leading edge. Figure 9 shows the final grid for the curved wall solution.

The results from this case are compared with the results from Arminjon and Madrane, ${ }^{11}$ whose results compare quite well to a collection of results from Müller

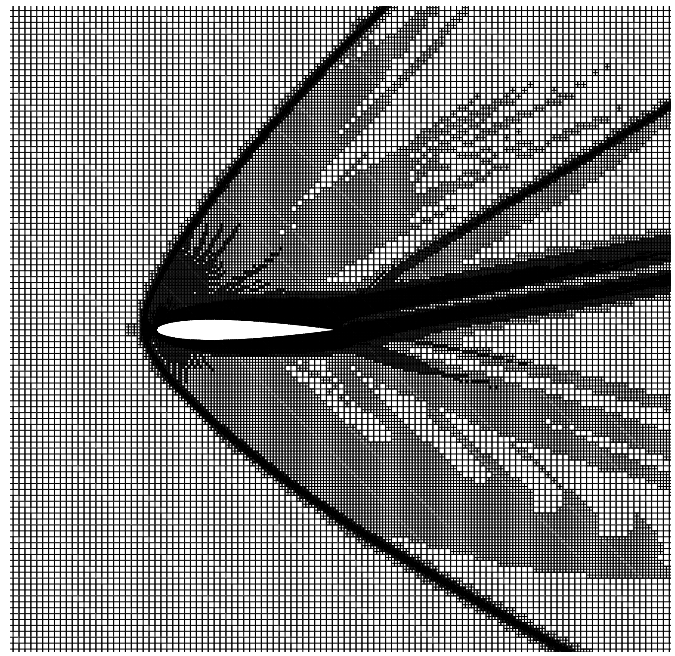

Fig. 9 Final Computational Domain for Supersonic Viscous NACA-0012 Flow

et al. ${ }^{12}$ from an international workshop on compressible Navier-Stokes solvers. The Arminjon and Madrane results are from an unstructured grid solution with 7962 vertices. The Müller results are from a structured grid solution with $257 \times 257(66,049)$ cells.

Figure 10 shows the surface pressure coefficient comparison between the NASCART-GT solutions and the results from Arminjon and Madrane. Both solutions generally show excellent agreement with the reference data. In general, there is nice agreement between both solutions and the reference data and no significant differences between the curved wall or flat wall solutions.

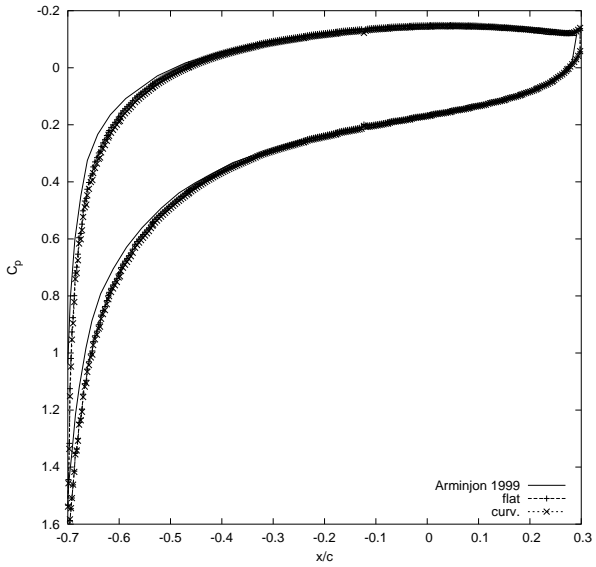

Fig. 10 Supersonic Viscous NACA-0012 Surface Pressure Coeffi cient

Figure 11 shows the skin friction coefficient for this case. This shows that again there are no large scale oscillations in the skin friction coefficient due to the varying surface cell sizes. There are, however smaller oscillations that are still apparent.

Figures 12 and 13 show the Mach contours for the flat wall and curved wall solutions, respectively. Figure 14 


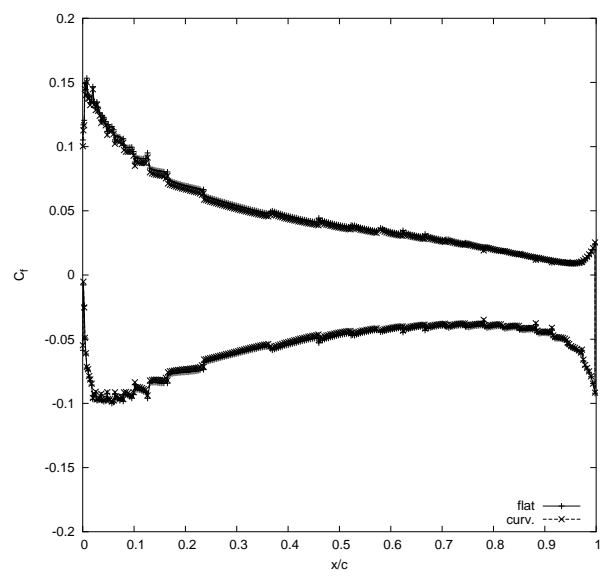

Fig. 11 Supersonic Viscous NACA-0012 Skin Friction Coeffi cient

shows the Mach contours from the Arminjon and Madrane reference. All three figures use a $\Delta M=0.1$ for the contours. Both wall boundary condition cases do an excellent job of capturing the flow features throughout the computational domain. The bow shock is crisply captured in both solutions.

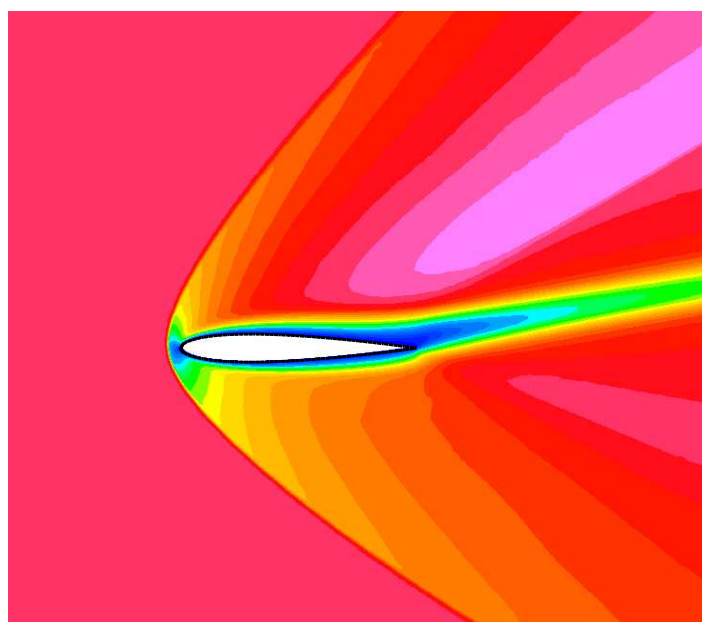

Fig. 12 Mach Contours for Supersonic Viscous NACA-0012 Flow Flat Wall

Finally, table 2 shows the lift and drag coefficients for the flat wall and curved wall cases. These results are compared to the Müller et al. reference mentioned above. The flat wall boundary condition slightly underpredicts the lift coefficient by $\mathbf{2 . 8 \%}$ compared to Müller et al. For the drag coefficient, the flat wall over-predicts both results by $6.8 \%$. The curved wall boundary condition under-predicts the results of Müller et al. by 3.1\%. For the drag coefficient, the curved wall boundary condition slightly over-predicts by $\mathbf{5 . 8 \%}$.

\section{Conclusions}

This research has provided insight into ways of extending the functionalities of Cartesian grid solvers into viscous effects modeling via novel boundary condition

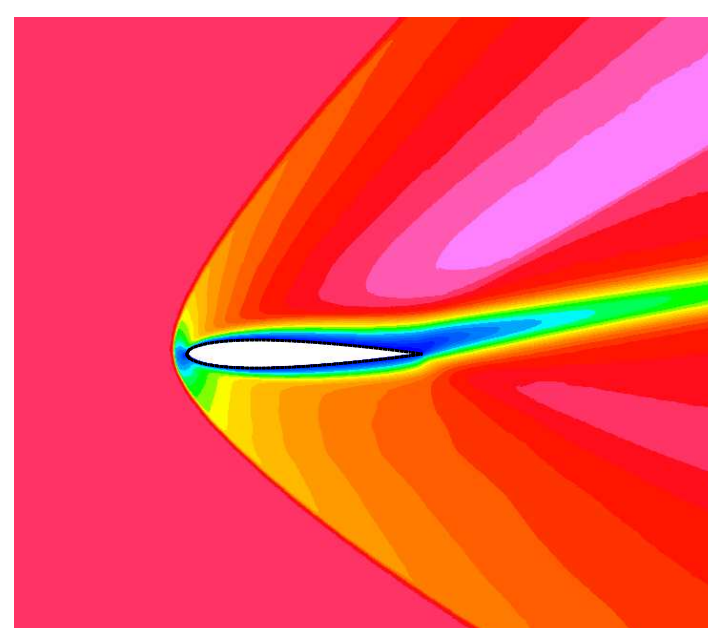

Fig. 13 Mach Contours for Supersonic Viscous NACA-0012 Flow Curved Wall

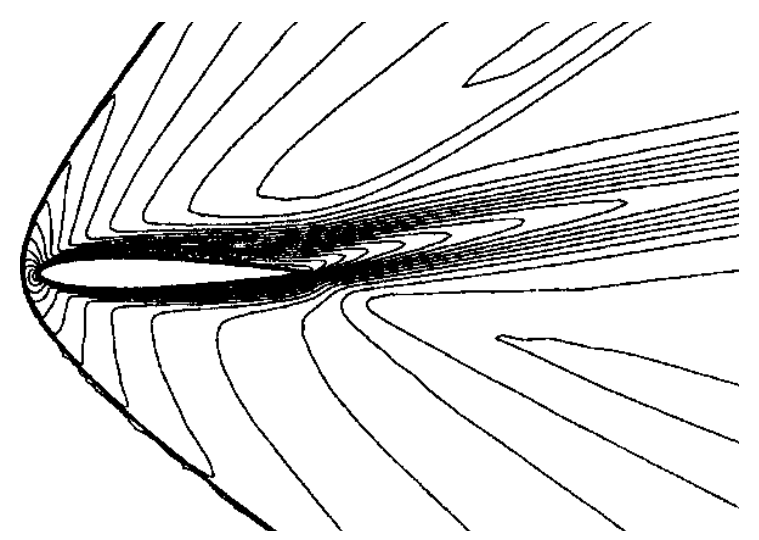

Fig. 14 Viscous Supersonic NACA-0012 Mach Contours from Arminjon and Madrane ${ }^{11}$

\begin{tabular}{cccc}
\hline & flat wall & curved wall & Müller $^{12}$ \\
\hline$C_{l}$ & 0.3292 & 0.3284 & 0.3388 \\
$C_{d}$ & 0.2685 & 0.2662 & 0.2515 \\
\hline
\end{tabular}

Table 2 Supersonic Viscous NACA-0012 Lift and Drag Results

treatments. The non-smoothness associated with the non-positivity of the viscous flux stencil for the surface cells have been greatly reduced in NASCART-GT by separating the surface cells from the finite volume formulation that is used to solve the rest of the computational domain. While the surface cells are not part of the finite volume formulation, their state is still determined by applying physically based conditions that are consistent with the boundary conditions associated with the surface. While this boundary condition treatment removes the formal conservative nature of the solver for these surface cells, preliminary experiments show that the non-conservativeness is very minimal. ${ }^{7}$

This new viscous solid boundary treatment developed for NASCART-GT removes the surface cells from the 
finite volume formulation in order to address the nonsmoothness and small time steps associated with the cut cell treatment. The state at the surface cells in determined by applying interpolation functions and the solid surface boundary conditions with either flat or curved wall approximations. This new treatment shows significant progress towards utilizing cut cell Cartesian grid methods for general bodies in viscous flows. In all cases presented, the interpolation formulations produce reasonable results without the non-smoothness problems associated with the stencil positivity in the viscous cases. The integrated quantities of lift and drag are well predicted with both the flat wall and curved wall boundary conditions, without significant improvements associated with the curved wall boundary conditions. The solid surface quantities compare well to existing results, with some cases showing difficulties near the leading edge. This difficulty is caused by the uniform surface cell size limitation imposed by the viscous scheme in order to avoid the viscous stencil positivity problem.

While, these results show significant improvements in the handling of viscous solutions on Cartesian grids, there are several areas of research that need to be examined further. In order to address the accuracy problems in the leading edge regions of the surface, the functionality of having multiple levels of refinement on the surface needs to be added to NASCART-GT. This needs to be carefully studied since Coirier showed nonsmoothness problems can arise even in regions where the cell sizes change is comparable to the changes at a refinement boundary. One possible approach to these surface refinement regions is to use a viscous flux reconstruction stencil based on the modified diamond-path Green-Gauss developed by Delanaye et al. ${ }^{13}$

\section{Acknowledgements}

David D. Marshall was supported by NASA's Graduate Student Research Program.

\section{References}

${ }^{1}$ Melton, J. E., Enomoto, F. Y., and Berger, M. J., "3D Automatic Cartesian Grid Generation for Euler Flows," 11th AIAA Computational Fluid Dynamics Conference, Orlando, FL, July 1993, AIAA-93-3386-CP.

${ }^{2}$ Berger, M. J. and LeVeque, R. J., "An Adaptive Cartesian Mesh Algorithm for the Euler Equations in Arbitrary Geometries," 9th AIAA Computational Fluid Dynamics Conference, Buffalo, NY, June 1989, AIAA-89-1930-CP.

${ }^{3}$ Melton, J. E., Berger, M. J., Aftosmis, M. J., and Wong, M. D., "3D Applications of a Cartesian Grid Euler Method," 33rd Aerospace Sciences Meeting and Exhibit, AIAA, Reno, NV, Jan. 1995, AIAA-95-0853.

${ }^{4}$ Aftosmis, M. J., Berger, M. J., and Adomavicius, G., "A Parallel Multilevel Method for Adaptively Refi ned Cartesian Grids with Embedded Boundaries," 38th Aerospace Sciences Meeting and Exhibit, AIAA, Reno, NV, Jan. 2000, AIAA-2000-0808.

${ }^{5}$ Coirier, W. J. and Powell, K. G., "Solution-Adaptive Cartesian Cell Approach for Viscous and Inviscid Flows," AIAA Journal, Vol. 34, No. 5, May 1996, pp. 938-945.

${ }^{6}$ Frymier, Jr., P. D., Hassan, H. A., and Salas, M. D., "Navier-Stokes Calculations Using Cartesian Grids: I. Laminar Flows," AIAA Journal, Vol. 26, No. 10, Oct. 1988, pp. 1181-1188.
${ }^{7}$ Marshall, D. D., Extending the Functionalities of Cartesian Grid Solvers: Viscous Effects Modeling and MPI Parallelization, Ph.D. thesis, Georgia Institute of Technology, Atlanta, GA, 2002.

${ }^{8}$ Marshall, D. D. and Ruffi n, S. M., "A New Inviscid Wall Boundary Condition Treatment for Embedded Boundary Cartesian Grid Schemes," 42nd Aerospace Sciences Meeting and Exhibit, AIAA, Reno, NV, Jan. 2004, AIAA-2004-0583.

${ }^{9}$ Casalini, F. and Dadone, A., "Computations of Viscous Flows Using a Multigrid Finite Volume Lamda Formulation," Engineering Computations, Vol. 16, No. 7, 1999, pp. 767-786.

${ }^{10}$ Bristeau, M. O., Glowinski, R., Periaux, J., and Viviand, H., "Presentation of Problems and Discussion of Results," Numerical Simulations of Compressible Navier-Stokes Flows, edited by M. O. Bristeau, R. Glowinski, J. Periaux, and H. Viviand, Notes on Numerical Fluid Mechanics, Friedr. Vieweg \& Sohn, 1987, pp. 1-40.

${ }^{11}$ Arminjon, P. and Madrane, A., "Staggered Mixed Finite Volume/Finite Element Method for the Navier-Stokes Equations," AIAA Journal, Vol. 37, No. 12, Dec. 1999, pp. 1558-1571.

${ }^{12}$ Müller, B., Berglind, T., and Rizzi, A., "Implicit Central Difference Simulation of Compressible Navier-Stokes Flow Over a NACA0012 Airfoil," Numerical Simulations of Compressible Navier-Stokes Flows, edited by M. O. Bristeau, R. Glowinski, J. Periaux, and H. Viviand, Notes on Numerical Fluid Mechanics, Friedr. Vieweg \& Sohn, 1987, pp. 183-200.

${ }^{13}$ Delanaye, M., Aftosmis, M. J., Berger, M. J., Liu, Y., and Pulliam, T. H., "Automatic Hybrid-Cartesian Grid Generation for High-Reynolds Number Flows around Complex Geometries," AIAA 37th Aerospace Sciences Meeting \& Exhibit, Reno, NV, Jan. 1999, AIAA-99-0777. 